Cahiers de recherche sociologique

\title{
La transition énergétique entre homéostasie du système et effondrement
}

Retour sur trois décennies de mobilisations discursives autour de

l'écologie

\section{Energy Transition, Between System Homeostasis and Collapse A Return on Three Decades of Discursive Mobilization on Ecology La transición energética entre la homeostasis del sistema y el colapso}

\section{El regreso sobre tres décadas de movilización discursiva alrededor de la ecología}

\section{Florence Rudolf}

Numéro 58, hiver 2015

Pour une sociologie de la transition écologique

URI : https://id.erudit.org/iderudit/1036205ar

DOI : https://doi.org/10.7202/1036205ar

Aller au sommaire du numéro

Éditeur(s)

Athéna éditions

ISSN

0831-1048 (imprimé)

1923-5771 (numérique)

Découvrir la revue

Citer cet article

Rudolf, F. (2015). La transition énergétique entre homéostasie du système et effondrement : retour sur trois décennies de mobilisations discursives autour de l'écologie. Cahiers de recherche sociologique, (58), 37-54.

https://doi.org/10.7202/1036205ar

\section{Résumé de l'article}

La transition énergétique prétend ouvrir un nouvel horizon dans le paysage français, mais cet horizon est-il à ce point inédit ? Pour en discuter, nous l'inscrivons dans une série dont elle fait partie. Par bien des aspects, les sémantiques qui rythment cette série partagent bien des points, dont le rejet de la figure révolutionnaire et l'affichage d'une certaine confiance dans la capacité de la modernité à faire face aux enjeux écologiques de la fin du $\mathrm{XX}^{\mathrm{e}}$ et du début du $\mathrm{XXI}^{\mathrm{e}}$ siècles. Par-delà cette similitude, le repérage des différences laisse entrevoir avec la référence à la transition un tournant plus menaçant qu'il n'y paraît au premier abord. Si la transition énergétique joue encore la carte de l'homéostasie, cette dernière est assombrie par le spectre d'un effondrement socio-écologique majeur qui s’annonce. 


\section{La transition énergétique entre homéostasie du système et effondrement Retour sur trois décennies de mobilisations discursives autour de l'écologie}

FLORENCE RUDOLF

\section{Introduction}

Après l'inflexion du progrès annoncé par le développement durable à la fin de la décennie 1980, la société française met un point d'honneur à entamer une transition énergétique sans précédent. Ce projet est adossé à un processus de dialogue et de propositions d'action, engagé en 2007, qui a abouti à la loi sur la transition énergétique, promulguée le 17 août 2015. Cette ambition a été activée par l'organisation de la COP 21 du 30 novembre au 11 décembre 2015. Par delà ce moment historique, cet engagement n'est pas un acte isolé à l'échelle européenne. Plusieurs États ont pris à bras le corps, chacun à sa façon et selon des ordres du jour propres, des réformes qui se voulaient ambitieuses en matière d'écologie. La transition énergétique, dont on notera après bien d'autres observateurs et commentateurs de notre état social qu'elle cultive l'ambivalence, poursuit les promesses des programmes qui l'ont précédée: relever les défis des enjeux écologiques sans casse. De la modernisation écologique - qui a pris davantage dans les pays d'Europe du 
Nord que ceux du Sud -, au développement durable, c'est d'une inflexion de la modernité plutôt que d'un changement de régime dont il est question.

Si cette intentionnalité est clairement revendiquée par l'expression de modernisation écologique qui se réfère explicitement à la modernité, voire par celle de développement durable qui s'inscrit également dans cette continuité par l'entremise de la référence au développement et au progrès, la filiation est moins nette quant à la transition énergétique. Ainsi que l'a encore rappelé récemment Ségolène Royale ${ }^{1}$, cette dernière évoque l'idée d'un passage d'un état à un autre. Selon cette lecture, la transition écologique joue explicitement avec la perspective d'un changement sociétal sans précédent. En même temps, elle place la croissance verte clairement dans sa feuille de route.

Cet affichage nécessite des explicitations. S'agit-il, pour reprendre l'interrogation de Lionel Charles ${ }^{2}$, d'un voyage plus ou moins orchestré, balisé, assuré ou d'une aventure plutôt risquée? Est-on dans l'ordre d'un processus qui suit son cours ou d'une expérimentation grandeur nature à haut risque? De quelle transformation est-il question en d'autres termes? S'agit-il de privilégier un changement d'état du «système», ou de tenter la substitution d'un «système» par un autre? Si la loi sur la transition énergétique laisse présager une transformation, sans équivoque, force est de constater que l'aboutissement de cette dernière demeure cependant indéterminé. Entre un réaménagement ou un changement de «régime», voire une mutation du «système», l'intervalle est large. Bien que sans ambiguïté quant à l'amplitude des possibles, la traduction concrète de la transition demeure obscure. L'absence de vision sociale et politique, susceptible d'indiquer des directions auxquelles se fier dans la construction de politiques publiques, soit d'un programme politique, n'est pas étrangère à cette indétermination. Cette dernière est loin d'être anodine du point de vue de la conception des politiques publiques et des pratiques sociales qui se réfèrent à la transition écologique et de leur évaluation. Le sens accordé au changement pèse sur la réception sociale de la transition écologique. De quel type de changement est-il question? À quelles échelles s'adresse-t-il?

$\mathrm{Si}$ tous ces questionnements trouvent à s'exprimer dans diverses scènes sociales et politiques, ils demeurent peu relayés dans l'espace public. Les nombreux documents édités pendant la période du projet de loi et qui ont alimenté les débats à l'Assemblée n'ont pas fait l'objet de publicisation dans l'espace public ${ }^{3}$. À aucun moment, même au cœur de l'événement, les orien-

1. La ministre actuelle de l'Environnement du gouvernement de Manuel Vals en France.

2. Lionel Charles, dans Helga-Jane Scwarwell, Divya Leducq, Anette Groux, Transitions énergétiques: quelles dynamiques de changement?, Paris, L'Harmattan, coll. « Colloques et rencontres», 2015.

3. Ecologie Les Verts (EELV) m'a remis gracieusement son dossier de presse au sommaire duquel figuraient: le scénario de Nega Watt, des dossiers d'Actu-environnement, de la Gazette des communes, de collectifs comme ATTAC ou encore de conférences de presses à l'initiative de différentes associations et EELV eux-mêmes. 
tations sociales exposées dans ces documents, n'ont été débattues dans l'espace public. Il n'y a pas eu la mobilisation sociale qu'on aurait pu en attendre compte tenu des enjeux que cette expression peut éveiller, en raison des utopies auxquelles elle est éventuellement associée. De manière générale, le projet de loi sur la transition énergétique est demeuré relativement confiné à un débat d'experts. Cette observation s'applique dans une moindre mesure à la conférence des parties sur le climat (COP 21), que le gouvernement veille à relayer, mais là aussi force est de constater l'atonie relative de l'espace public. Atonie qui a été redoublée par les attentats de novembre 2015 qui, après avoir meurtri la société, ont porté atteinte aux réjouissances que préparaient les associations et la société civile en vue de cet événement. Ce constat réitère l'urgence d'investir de larges scènes publiques, ouvertes au plus grand nombre, dans l'intérêt de la démocratie.

À défaut de pouvoir se prononcer sur l'impact sociétal qu'aura la transition énergétique sur la durée, il est en revanche possible et souhaitable de réfléchir au type d'interrogations et de réflexions qu'actualise un tel projet. Une rétrospective consacrée aux discours qui accompagnent l'entrée en politique de l'écologie peut offrir un tel point de vue. Nous procédons à cette dernière par rapport à la série discursive dans laquelle s'inscrit la transition écologique de manière à mettre en évidence l'imaginaire dont procèdent les sémantiques écologiques qui accompagnent la modernité tardive depuis la fin du $\mathrm{XX}^{\mathrm{e}}$ siècle. L'investigation que nous menons s'organise davantage autour de considérations relatives à la capacité de la modernité à se réformer et au caractère révélateur de l'écologie de ce point de vue qu'en référence à des recompositions sociales par l'entremise de l'écologie. Notre enquête procède d'une observation participante au sein de cercles universitaires et non d'une inscription dans les organisations politiques et sociales qui se saisissent de ces enjeux. Il s'agit donc d'une analyse à distance des mobilisations sociales proprement dites. Une des conclusions majeures de cette exploration a trait à l'ambivalence lancinante à l'égard de la crise écologique qui se dégage de l'analyse de ces sémantiques. Ce constat est significatif d'un état général de notre constitution sociale. Il conforte les diagnostics consacrés à la modernité avancée en termes d'action et d'engagement notamment.

\section{Des programmes au crible d'une analyse sémantique}

La transition énergétique s'inscrit dans des discours qui s'organisent en réponse à la crise politique: elle dessine un programme politique qui prend le relais d'autres programmes qui se sont succédé avant elle depuis que l'éco- 
logie est entrée en politique 4 . Son inscription dans cette chronologie invite à s'interroger sur le sens de cette succession sémantique. Quel rapport ces différents programmes entretiennent-ils entre eux et qu'est-ce qui justifie, si de justification on peut parler, ce renouvellement sémantique? Cette interrogation structure notre propos.

Qu'est-ce qui relie la modernisation écologique au développement durable et à la transition énergétique du point de vue de notre constitution sociale et de la prise en charge de l'écologie? La perspective d'une sortie de crise sans changement? Cette impression est d'un grand intérêt car elle attire l'attention sur une des constantes des réponses à la crise écologique depuis les années 1970: la conception de programmes porteurs d'une transformation progressive sans souffrance et dont tout le monde sortirait gagnant ${ }^{5}$. Si de bouleversements il est in fine question, ces derniers doivent avoir lieu en sourdine, passer quasi inaperçus, n'être remarquables que rétrospectivement.

\section{La modernisation écologique}

Le premier scénario, désigné de modernisation écologique, est une invention à mi-chemin entre un imaginaire sociologique et de décideur qui procède pleinement de la critique sociale du développement, popularisé par le président Truman en 1949 pour relancer l'économie mondiale, et qui est contemporaine de l'invention du développement durable. Arthur Mol, un de ses principaux inventeurs, revenait sur les conditions d'émergence de cette expression dans un séminaire consacré à une rétrospective sur la modernisation écologique à Freiburg en Brisgau en 2013. Il rappelait à cette occasion que la modernisation écologique était une sorte d'hybride entre deux types de communauté et d'activité ${ }^{6}$. Les registres mobilisés par l'expression de modernisation écologique en témoignent très explicitement: la convocation de la modernité et de la modernisation fait écho aux théories sociologiques, mais également à un imaginaire post-Seconde Guerre mondiale dans lequel le progrès, incarné par la reconstruction de l'économie mondiale, hante les esprits et guide les politiques. La référence à l'écologie relaye quant à elle les revendications sociales, en particulier de la jeunesse, en faveur d'une démo-

4. Bruno Latour, Politiques de la nature. Comment faire entrer les sciences en démocratie?, Paris, La découverte, 1999.

5. La récurrence du «gagnant, gagnant» a de quoi irriter et éveiller la suspicion. Comme s'il était soudainement possible d'assurer une redistribution des profits alors que la rareté de certaines ressources exacerbe les convoitises.

6. Florence Rudolf, «De la modernisation écologique à la résilience: un réformisme de plus?», VertigO, La revue électronique en sciences de l'environnement, vol. 13, n³, décembre 2013. 
cratisation de la société en réponse au mode d'organisation disciplinaire ${ }^{7}$ qui perdure dans les Trente Glorieuses ${ }^{8}$.

Le cap pris par la modernisation à marche forcée dans les années de reconstruction après-guerre montre des essoufflements. Ces derniers trouvent différents canaux d'expression, dont l'aspiration à la démocratie et la conscience de la finitude du monde. Ces deux motifs, situés historiquement par la révolution de 1968 et par le premier choc pétrolier daté de 1971, ne convergent pas nécessairement. Leur articulation demeure suspendue à l'aptitude des sociétés civiles d'y veiller. À compter des années 1970, la rareté, qu'elle soit associée à la perspective d'un pic pétrolier, hante de manière récurrente les réflexions des philosophes et sociologues des techniques, des intellectuels et différents experts en développement et en économie, mais aussi des entrepreneurs éclairés avec plus ou moins d'acuité selon l'actualité. Cette thématique alimente la sortie nécessaire des économies fondées sur des énergies fossiles, mais aussi l'invention de nouvelles formes de développement qui peuvent converger avec le désir de démocratie. Si l'épuisement d'un monde fini retient l'attention et anime les esprits, ce n'est pas l'unique source de mobilisation sociale de l'époque. La généralisation d'un mode d'organisation rationnel et autoritaire du travail à l'ensemble de la société en passant par la reconstruction mortifère des villes, dont la désaffection des grands ensembles est un indicateur parmi d'autres, est également au cœur de la critique sociale.

Il est donc question des limites de la croissance, notamment par l'entremise de la finitude des ressources en pétrole et de la planète, mais aussi de processus d'entre-capture systémiques, hérités d'un faux «contrat social» ou «système» qui impose son carcan sur l'immense majorité de la société. Le développement dévoyé ${ }^{9}$, véritable cheval de Troie du capitalisme ${ }^{10}$, poursuit un processus dévastateur et inégal qu'il convient d'inverser. Ce renversement prendra des accents révolutionnaires ou réformistes, selon qu'il est question ou non de préserver le projet de la modernité. Si la thématique de la finitude est bien au rendez-vous, l'époque est aussi à l'effervescence: elle rêve de liberté, de participation et de partage des ressources. Les mobilisations sociales post-soixante-huit, dont les effets se font sentir bien au-delà du mois de mai, s’organisent en réponse au caractère disciplinaire de la modernité, au prix d'un rejet de cette dernière ou au contraire au nom de son esprit. Sans

\footnotetext{
7. Peter Wagner, Liberté et discipline. Les deux crises de la modernité, Paris, Métailié, 1996.

8. Une jeunesse allemande, documentaire réalisé par Jean-Gabriel Periot, 2015.

9. Richard B. Norgaard, Development Betrayed: The End of Progress and a Co-evolutionary Revisioning of the Future, Londres, Routledge, 1994.

10. Gilbert Rist, Le développement. Histoire d'une croyance occidentale, Paris, Presses de Sciences Po, 2007.
} 
poursuivre davantage cet effort de rétrospective qui procède toujours un peu d'une reconstruction mythique et de l'art de la fiction, il nous semble que l'invention de la modernisation écologique est indissociable de cet horizon de significations où finitude et aspiration à un processus de démocratisation cohabitent. Cette cohabitation prenant diverses modalités d'action et de transformation selon les forces sociales et politiques qui s'en emparent.

L'invention de la modernité écologique porte la marque du réformisme, elle affiche sa confiance en la modernité, dont elle envisage l'avenir à travers le concours des différentes institutions modernes. Selon elle, ces dernières sont en mesure de relever le défi de la crise écologique par l'association de la science et de la technique au service d'une économie de marché encadrée, c'est-à-dire régulée par l'État et les politiques environnementales, dans une constitution démocratique. À cet égard, la modernisation écologique fait écho aux travaux de Jürgen Habermas, dont la thèse de l'agir communicationnel, qui datent de la même époque, où il est question de la modernité comme d'un projet inachevé, sous-entendu qui se doit d'être poursuivi et accompli. Outre cette inscription constitutionnelle, la modernisation écologique travaille à l'élaboration de processus de modernisation plus attentifs à la fragilité des équilibres entre «nature» et "société». C'est une expression qui se veut rassembleuse et fédératrice: une sorte de nouveau pacte social, permettant de concilier économie et écologie, voire de les réconcilier. Cette formule pacificatrice, qui n'a pas été sans soulever des résistances, notamment des forces sociales et politiques hostiles au système capitaliste, voire au développement et à la modernité qui semblaient inféodés à ce dernier, a fini par s'imposer par l'entremise de la figure du compromis, cette dernière étant également au cour du développement durable ${ }^{11}$.

\section{Le développement durable}

Le développement durable procède d'un imaginaire qui n'est guère éloigné de celui de la modernisation écologique, ne serait-ce qu'en raison de la référence explicite qu'il fait au progrès par l'entremise du développement. Il semble cependant épouser une cause plus élargie que la modernisation écologique par les références qu'il établit avec la gouvernance et la dimension sociale et culturelle de son programme d'une part, et de sa prétention à la globalisation, d'autre part. Alors que la modernisation écologique apparaissait davantage comme un programme du «Nord", à l'intention des nations anciennement industrialisées, une sorte de défi en termes d'innovations tech-

11. Corinne Gendron, Le développement durable comme compromis. La modernisation écologique de l'économie à l'ère de la mondialisation, Québec, Presses de l'Université du Québec, coll. «Pratiques et politiques sociales et économiques», 2006. 
nologiques et institutionnelles, le développement durable aspire à s'imposer comme un programme destiné à l'ensemble de la planète. À titre de projet onusien, il se présente en 1987 comme une entreprise de pacification, voire de réconciliation, dans un monde clivé. La chute du mur de Berlin et du bloc de l'Est conférera un crédit robuste à la mystification d'un monde engagé dans une dynamique commune dont le développement durable est le messager. Il renoue à cet effet avec le caractère mystificateur et réducteur du discours sur le développement, au sortir de la Seconde Guerre mondiale.

Au regard de l'imaginaire du développement et de la modernisation dite du "premier ordre ${ }^{12}$ ", mais également de la modernisation écologique, on ne peut exclure cependant que l'invention du développement durable réalise une montée en complexité par rapport à ses prédécesseurs. Par les synergies entre économie, écologie et société qu'il préconise, il prend de la hauteur par rapport à l'hybridation de l'économie et de l'écologie qu'impulse la modernisation écologique. L'ambition par rapport à la complexité, dont il convient de relever les défis, n'est pas au goût de tous les acteurs engagés dans la cause écologique ${ }^{13}$, en raison d'un risque d'affadissement de cette dernière.

Le développement durable n'a pas transigé sur cette exigence ainsi qu'en témoigne l'intégration de la culture, quatrième pilier à ajouter à sa carrière à l'occasion du sommet de Durban en 2004. La référence à la culture, que cette dernière soit pensée au sens restreint de culture politique, soit de gouvernance, ou dans un sens élargi, accroît encore les exigences du développement durable en matière de complexité. La culture peut être envisagée comme l'opérateur ou la force motrice qui assure la formation de synergies entre économie, écologie et société et en garantit la diversité et la créativité. L'implication de la culture crée, selon cette lecture, un espace pour la démocratie de proximité, voire pour la reconnaissance des peuples et de leur droit à des chemins spécifiques de développement. La prise en compte de la dimension culturelle peut aller jusqu'à être envisagée comme un ethos spécifique au même titre que Max Weber entendait l'implication de ce dernier dans l'essor du capitalisme ${ }^{14}$, ou plus récemment à la manière dont les

12. Ulrich Beck, La société du risque. Sur la voie d'une autre modernité, Paris, Aubier, 2001.

13. Notamment des Fundis au sein des Grünen (Les Verts allemands) en référence à leur fondamentalisme qui les conduisait à refuser toute concession et alliances avec d'autres forces sociales contrairement aux Realos qui incarnaient les pragmatiques avant l'heure (Florence Rudolf, «Économie et écologie: les politiques environnementales de l'Allemagne contemporaine», dans Claire Demesmay, Hans Stark (dir.), Qui dirige l'Allemagne?, Villeneuve d'Ascq, Presses Universitaires du Septentrion, 2005, p. 213-227).

14. Max Weber, L'éthique protestante et l'esprit du capitalisme (1904-1905), traduction par Jacques Chavy, Paris, Plon, 1964 (traduction par Isabelle Kalinowski, Paris, Flammarion 2000; Paris, Gallimard, 2003). 
coordonnateurs du rapport Culture in, for and as Sustainable Development ${ }^{15}$ la considérent.

Sur le plan d'une pure rhétorique, le développement durable présente bien des atouts, même par rapport à la figure du progrès, guide par excellence de la modernité. S'il ne rompt pas avec cette dernière, il la questionne, en réhabilitant l'idée de chemins de développement. S'il semble toujours légitime pour les sociétés de viser le progrès, ce dernier a perdu de son univocité $^{16}$. Contrairement à la modernisation écologique qui est une invention de chercheurs principalement d'Europe du Nord et dont se sont inspirées les politiques de ces États, le développement durable s'est imposé par les conférences internationales de facture onusienne et par leur transposition à différentes échelles, dont l'échelle des réseaux de villes, innovation notable en la matière ${ }^{17}$. $S^{\prime}$ il demeure une référence incontestée et non incontestable, il s'est vu progressivement relayé par des dossiers plus explicites dont les changements climatiques et l'effondrement de la biodiversité. Cette évolution est vraisemblablement imputable au caractère un peu abstrait de cette «idée régulatrice» de la modernité tardive.

\section{Les changements climatiques, principaux vecteurs de la transition énergétique}

Les changements climatiques vont progressivement prendre le relais du développement durable, dont l'opérationnalité s'avère à la pratique contestable. Ils le prennent à titre de risque global majeur qui menace les équilibres géopolitiques planétaires. Si le développement durable offre de multiples traductions à différentes échelles territoriales et en termes de responsabilité d'acteurs, son principal travers demeure son inconsistance quant aux risques qu'il cible précisément. Le développement durable pêche par cette formidable aptitude à des déclinaisons en listes interminables d'actions, du type d'un inventaire à la Prévert, dont de nombreux Agendas Locaux 21 sont une illustration. Ce foisonnement est imputable à la difficulté du développement durable à faire système, c'est-à-dire à converger autour de variables ou logiques propres clairement identifiables. Le développement durable serait-il rétif à l'esprit de système? S'il invite aux synergies, il ne converge pas pour autant autour d'un codage propre

15. Joost Dessein, Katrina Soini, Graham Fairclough, Lummina Horlings (dir.), Culture in, for and as Sustainable Development. Conclusions from the cost action 151007. Investigating cultural sustainability, European Cooperation in Science and Technology, Jyväskylä, Finland, Jyväskylä University Press, 2015.

16. Encore qu'il conviendrait de s'interroger jusqu'à quel point la figure univoque du progrès prend plusieurs visages, dans l'imaginaire du développement durable, ou si ce sont seulement les sentiers susceptibles d'y conduire qui peuvent diverger?

17. Kristine Kern, "Climate Governance in the European Union multilevel system: the role of cities», dans Inger Weibust, James Meadowcroft (dir.), Multilevel environmental governance. Managing water and climate change in Europe and North America, Cheltenham, Edward Elgar, 2014, p. 111-132. 
à l'organisation systémique. Ce trait de caractère pose apparemment problème du point de vue de la conception des politiques publiques.

L'avantage des changements climatiques tient à son couplage aux questions énergétiques, sans renoncer aux enjeux de complexité et de globalité inhérents à notre temps et que le développement durable ${ }^{18}$ s'est employé à relever. Dans le cas des changements climatiques, ces derniers offrent une traduction opérationnelle explicite et cohérente par l'entremise de la question énergétique avec laquelle ils sont structurellement couplés. Les changements climatiques étant l'expression d'un déséquilibre énergétique par la libération accrue d'énergies fossiles extraites du sol depuis l'ère industrielle ${ }^{19}$.

L'entrée par l'énergie peut se révéler d'une efficacité remarquable en termes de stratégies parce que transversale à l'ensemble des activités et domaines de la vie sociale ${ }^{20}$. Les choix énergétiques peuvent être appréhendés à travers la production de gaz à effet de serre mais également comme l'expression de rapports sociaux inégau ${ }^{21}$. La transition énergétique est susceptible d'interroger les répercussions de choix énergétiques à différentes échelles de l'aménagement des territoires jusqu'aux questions de géopolitiques les plus sensibles. À ce titre, elle n'est pas vouée à rester cantonnée à une dimension technique et besogneuse et peut très bien nourrir de grandes ambitions politiques. Elle peut prétendre à une certaine radicalité en ce que les substitutions et modifications opérées à son niveau impliquent des répercussions dynamiques susceptibles d'avoir des effets de résonance conséquents et durables. La transition énergétique n'est donc pas condamnée à rester cantonnée à des préoccupations strictement comptables, elle peut contribuer à la déconstruction d'interdépendances non vertueuses. En bref, elle peut se décliner en compétences techniques, susceptibles de diffuser dans tous les domaines de la vie, ainsi qu'en stratégies politiques, sociales et économiques sujettes à controverses et polémiques. À cet égard, le couple changements climatiques, transition énergétique présente de nombreux avantages, dont le fait de relativiser la vision consensuelle de l'écologie comme intérêt bien compris et partagé par tous, voire de la démentir ${ }^{22}$. Les changements clima-

18. Florence Rudolf, «La réception territoriale du changement climatique ou comment le changement climatique contribue à l'émergence de territoires et politiques climatiques spécifiques», dans P. Boudes, M. Colombert (dir.), Adaptation aux changements climatiques et trames vertes: quels enjeux pour la ville?, VertigO, La revue électronique en sciences de l'environnement, Hors Série 12, mai 2012.

19. Jean Poitou, Pascale Braconnot, Valérie Masson-Delmotte, Le climat: la terre et les hommes, Les Ulis, EDP Sciences, coll. «Une introduction», 2014.

20. Alain Gras, Le choix du feu. Aux origines de la crise climatique, Paris, Fayard, 2007.

21. Helga-Jane Scarwell, Divya Leducq, Annette Groux (dir.), Réussir la transition énergétique, Lille, Presses universitaires du Septentrion, 2014.

22. Florence Rudolf, Le climat change... et la société?, Montreuil-sous-Bois, La ville brûle, 2009. Lionel Charles, "Adaptation au changement climatique et sciences sociales», Revue Pollution Atmosphérique. Climat, Santé, Société, Numéro Spécial, juin 2013, p. 96-105. 
tiques mettent en scène des territoires prédateurs et des territoires sacrifiés et annoncent un climat social plus tendu ainsi qu'en témoigne le réseau pour la justice climatique. Les changements climatiques soulèvent des questions de solidarités intra et intergénérationnelles, avec une dimension géopolitique plus exacerbée que le développement durable et a fortiori que la modernisation écologique.

La transition énergétique, associée aux changements climatiques, ouvre donc une nouvelle page de la mise en récit de la crise écologique qui renoue avec un des fondements majeurs de la crise écologique des années 1970. À la différence de la perspective du pic pétrolier et donc de la rareté, l'effet de serre constitue un nouveau risque qui redouble la crise écologique par la perspective d'un dérèglement majeur du système planétaire. Cette nouvelle menace est aujourd'hui rassemblée sous l'expression d'anthropocène ${ }^{23}$. Selon cette dernière, l'humanité est érigée en nouveau facteur écologique susceptible de contribuer à un effondrement en cascade, affectant par conséquent tant les différents écosystèmes que les systèmes sociaux actuels.

En dépit de ces perspectives qui valent d'arguments pour une mobilisation sans précédent, l'engouement pour la transition énergétique ne semble pas être au rendez-vous. À quelles réserves associer cette réception mitigée? Une des raisons principales de ces dernières nous semble inscrite dans l'histoire dont la transition énergétique participe, soit à la répétition du même ou au sentiment de la répétition du même. Certes, si rien ne s'oppose en principe pour que les changements climatiques et la transition énergétique se renforcent mutuellement, c'est l'engagement économique, politique et social qui sous-tend cette convergence et ces synergies qui demeure problématique. Quelle valeur accorder aux protagonistes d'une cause qui ne parviennent pas à se présenter, que ce soit en images ou en mots? Comment créditer une cause qui ne parvient ni à faire récit ni à faire émerger une vision, même floue de ce vers quoi elle tend?

La sémantique de la transition écologique s'affirme dans un contexte contrasté par rapport à ceux qui ont vu naître la modernisation écologique et le développement durable. Alors que la crise écologique des années 1970 pouvait encore apparaitre comme une crise inédite, susceptible de stimuler l'intelligence et la créativité de la modernité, l'enlisement de la crise écologique dans la durée, associé au crack boursier de 2008, incite à un retour critique sur ces interprétations généreuses. Si la modernisation écologique avait pu se profiler comme un projet susceptible d'encadrer l'économie capitaliste par les inno-

23. Christophe Bonneuil, Jean-Baptiste Fressoz, L'événement anthropocène. La terre, l'histoire et nous, Paris, Seuil, 2013. 
vations écologiques et sociales qu'elle relayerait, cette promesse a perdu de sa vraisemblance de nos jours. Que s'est-il passé?

\section{Des sémantiques en perte de sens et de crédibilité}

Le discrédit qui pèse sur ce récit est indissociable de la fragilisation de l'Étatprovidence. Pour que l'équation de la modernisation écologique soit tenable, il faut que les marchés soient encadrés et qu'une partie du capital soit redistribuée aux plus faibles par l'investissement dans des services publics notamment. En bref, il faut une instance politique - l'État ou pourquoi pas des gouvernements urbains ou régionaux - susceptibles de garantir une certaine pacification des rapports sociaux et leur orchestration ${ }^{24}$. Si ce rôle semblait assuré par les États-providence occidentaux fortement intégrés, la situation n'est plus aussi simple depuis la crise économique de 2008. Conformément à l'effet boomerang, décrit par Ulrich Beck dans La société du risque ${ }^{25}$, l'illusion d'une redistribution des profits a fini par éclater au grand jour. Cette mystification a profité pendant quelques décennies des progrès enregistrés à l'échelle des États-Nations européens, certains plus que d'autres, certaines régions mieux que d'autres ${ }^{26}$. Les progrès enregistrés par l'édiction de lois sur l'environnement, entérinés par les investissements effectués par les entreprises en faveur de la qualité environnementale, sans affecter le pacte social, dans certaines régions du monde, ont laissé complètement dans l'ombre l'état de l'environnement et des rapports sociaux dans d'autres régions. Les tiers qui étaient susceptibles de garantir une certaine pacification sociale connaissent une fragilisation effective à mesure que les marges de profit s'amenuisent. Parallèlement à la progression de cette vulnérabilité, il convient de tenir également compte des coûts croissants de la crise économique et écologique. Les États et les gouvernements seront de plus en plus sollicités pour venir "éteindre le feu», assurer des fonctions de police et de pompiers. Ils seront de plus en plus en porte-à-faux par conséquent avec une partie de la population. Le discrédit retentit sur les tenants de la modernisation, qu'elle soit affublée du qualificatif de social-démocratie et d'écologie: classe politique, professionnels et experts qui tentent désespérément ou mollement de lutter contre cette dégradation de la condition sociale et écologique. Face à cette débâcle, les voix des extrémistes gagnent en puissance et en pouvoir

24. Rasmig Keucheyan, La nature est un champ de bataille. Essai d'écologie politique, Paris, La découverte, coll. «Zones», 2014.

25. Ulrich Beck, op. cit.

26. Même à l'échelle européenne, les contrastes entre régions demeurent et attestent de ce que le rattrapage des régions sous-développées est un argument fallacieux. 
de conviction. La modernité qui pouvait être créditée de nombreux atouts ressort fragilisée de ces épreuves.

À ces éclairages, il convient d'ajouter les effets de la mondialisation. Tant que les bilans étaient tirés à des échelles locales sans tenir compte de l'état général de la planète, la dynamique impulsée par la modernisation écologique pouvait paraître prometteuse. À partir du moment où l'évaluation du programme intègre l'état de la planète dans son ensemble, les résultats s'avèrent moins concluants, voire franchement désastreux. Pourquoi? Tout simplement parce que la modernisation écologique n'est pas parvenue à pacifier des rapports sociaux de plus en plus tendus en régime de dérégulation du capitalisme. Elle se trouve même suspectée de contribuer à cette dynamique en garantissant de nouvelles niches et de nouveaux marchés aux investissements financiers, dans le domaine des services écologiques et climatiques notamment.

Parmi les critiques les plus saillantes formulées à l'encontre de la modernisation écologique ${ }^{27}$, on retiendra que cette dernière n'a permis au mieux que de temporiser le problème, c'est-à-dire de faire gagner ou perdre du temps selon les points de vue adoptés, c'est-à-dire selon les intérêts en présence. La temporisation équivaut à une stratégie sociale opposée aux formes de politisation. Elle garantit une certaine opacité sociale par déficit de publicisation de sorte à laisser les rapports sociaux jouer «librement», c'est-àdire en coulisse, et œuvrer en faveur de dénouements qui n'auront pas fait l'objet de débats politiques et de polémiques sociales ${ }^{28}$. Du point de vue des «gagnants ", c'est-à-dire des groupes sociaux qui ont tout à gagner du maintien du système actuel, ou tout simplement de ceux qui craignent que le changement n'engendre le chaos, la temporisation est la solution la moins intolérable. Quant aux populations les plus vulnérables, ce gain de temps correspond à une fragilisation de leur condition car toute poussée du capitalisme ou du néolibéralisme équivaut à une destruction des niches encore épargnées et où ces dernières pouvaient trouver refuge. Les fameuses formules du type «faire rimer l'économie avec l'écologie» ou du «gagnant, gagnant", promues par la modernisation écologique, apparaissent de plus en plus comme des leurres destinés à endormir les populations.

Ces critiques intensifient les exigences à l'encontre de la transition énergétique. Dans quelle mesure la transition énergétique assume-t-elle cette historicité et comment? Cette dernière est attendue au tournant: elle est condamnée à réussir au risque d'entretenir le sentiment que les programmes politiques

27. Florence Rudolf, «De la modernisation écologique... », op. cit.

28. Florence Rudolf, «Les formes de conflictualité associées à la société du risque», dans Myriam Klinger et Sébastien Schehr, Lectures du conflit, Strasbourg, Néothèque, 2010, p. 279-292. 
élaborés en réponse à la crise écologique s'organisent davantage autour de la sauvegarde d'un système, celui d'une économie capitaliste qui s'emballe, qu'au profit d'une modernité en quête d'une constitution écologique et sociale, c'est-à-dire solidaire.

Sans décliner davantage les espoirs que ces programmes, inventés pour faire face aux enjeux écologiques depuis les années 1970, ont pu alimenter, c'est du pouvoir d'action des sociétés sur elles-mêmes, voire de l'humanité sur son histoire, dont il est question en creux à travers ces récits qui s'interpellent et s'affrontent. Il n'est pas inutile de rappeler que la modernisation écologique s'est imposée dans les années 1980 contre le scénario de la transformation radicale de la société, classiquement identifiée de révolutionnaire ${ }^{29}$. Les protagonistes d'une forme de réformisme du système qu'étaient les modernisateurs avançaient de solides arguments comme l'évitement de la violence d'une part, et le fait qu'aucun nouveau système ne saurait émerger comme par miracle d'un champ de ruines, d'autre part.

Qu'en est-il de ces arguments aujourd'hui? Qu'en est-il de la réception du modèle du changement incrémental? Est-il encore crédible ou résonnet-il comme une entente dévoyée, un «faux» contrat social? Quelles alternatives s'offrent a contrario pour contrer des alliances factices et insoutenables? Toutes ces interrogations ne sauraient trouver des réponses dans une analyse de discours en quête de leur agentivité potentielle. Elles nous interpellent à titre d'acteurs susceptibles de se constituer en publics pour infléchir la flèche du temps.

En l'état, l'émulation en faveur de la transition énergétique demeure timide si l'on s'en tient à des indicateurs comme la publicisation de la transition énergétique dans l'espace public ou comme l'état de l'opinion publique, si tant est que cet indicateur puisse avoir une pertinence ${ }^{30}$. En raison des limites propres à ces outils, il n'y a pas grande matière à exploiter de ces observations, sinon que l'état des communications généralisées de la transition énergétique demeure extraordinairement faible. En se tournant vers des publics spécifiques ou des scènes constituées, la situation apparait moins atone. Par l'entremise des pouvoirs et fonds publics, de nombreuses initiatives voient le jour, même avec les milieux des entreprises en association avec leurs représentations syndicales ou consulaires ${ }^{31}$. Ces partenariats s'organisent autour de projets qui, à l'instar de l'économie circulaire ou de l'écologie industrielle, font état d'une recherche action ajustée à la transition énergétique et aux changements climatiques. Peut-on pour autant annoncer que la transition au sens d'un passage

29. Florence Rudolf, «De la modernisation écologique... », op. cit.

30. Pierre Bourdieu, Questions de sociologie, Paris, Éditions de Minuit, 1984.

31. ANR SECIF, coordonnée par Pascale Braconnot (2011-2014). 
d'un état à un autre soit engagée? Gardons-nous de tels pronostics et limitons nous tout au plus au constat qu'un certain nombre d'acteurs - collectivités, associations, entreprises, acteurs financiers -, observent le phénomène, voire se positionnent en prévision de cette transition, dont le cap demeure incertain.

\section{Entre homéostasie systémique et effondrement?}

En tout état de cause et comme Max Weber nous y invite dans ses conférences sur le savant et le politique ${ }^{32}$, l'adoption de la posture de l'observation invite à la prudence. S'il semble préférable de s'abstenir de tout pronostic ${ }^{33}$, la présente rétrospective sur quelques décennies fait tout de même état d'une incroyable aptitude des systèmes à leur préservation ${ }^{34}$. Cette affirmation n'exclut pas pour autant des déplacements et des glissements qui méritent d'être observés et relevés d'une sémantique à une autre.

Au compte du premier argument, soit de l'incroyable résilience du système de la société, c'est vers Niklas Luhmann, sans conteste et hésitation, que nous nous tournons pour rendre compte de l'homéostasie du système en dépit de plusieurs décennies de mobilisations écologiques. Luhmann passe pour un des sociologues du $\mathrm{XX}^{\mathrm{e}}$ siècle qui, en dépit de sa réputation de conservateur, a sans doute le plus contribué à révolutionner la sociologie classique. Il s'y est employé à partir d'une relecture de la théorie des systèmes de Parsons qui emprunte in fine davantage aux sciences de la vie ${ }^{35}$ et plus précisément à Maturana et Varela. Une des contributions majeures de Luhmann pour la question qui nous intéresse ici tient à l'interprétation qu'il propose de la réception des programmes politiques inventés en réponse à la crise écologique sur plusieurs décennies. À l’instar de tous les événements communicationnels qui participent de l'autopoièse sociale, soit du système de la société comme système autopoiétique structuré autour du sens, ces derniers procèdent à des irritations dans l'environnement des différents systèmes de la société. À aucun moment, on ne peut parler en conséquence d'interventions des acteurs sur la société et a fortiori sur le monde out there ${ }^{36}$, mais uniquement de l'impact du sens structuré en communications sociales sur des sous-systèmes de la société qui résultent eux-mêmes de la structuration

32. Max Weber, Le savant et le politique, Paris, Plon (1964), 1990. L'éthique protestante et l'esprit du capitalisme, Paris, Plon, coll. 1018 (1re édition 1959).

33. Alain Gras, Sociologie des ruptures. Les pièges du temps, Paris, PUF, 1979.

34. Joseph Heath, Andrew Potter, Révolte consommée. Le mythe de la contre-culture, Paris, Éditions Naïve, 2005.

35. Niklas Luhmann, Soziale Systeme, Frankfurt/Main, Suhrkamp, 1984. Florence Rudolf, «Niklas Luhmann, une théorie de la vie adaptée à la sociologie», Sociétés, n 43, 1994, p. 29-41

36. Si tant est que cette expression de monde out there fasse sens chez Luhmann. L'idée de monde suppose une organisation, une entité du type système autopoiétique qui n'est pas approprié, d'où le recours au concept d'environnement, ce dernier formant un horizon de possibles et non une entité en soi. 
du sens. Les conséquences de ces recompositions pouvant in fine avoir des répercussions sur des agencements plus concrets et tangibles.

Il y aurait beaucoup de choses à dire sur cette approche, dont des réserves à formuler ${ }^{37}$, mais ce n'est pas le lieu d'entrer dans cette discussion. La transposition de cette version du paradigme systémique à la modernisation écologique et à la transition énergétique permet de considérer ces politiques sous l'angle de communications généralisées susceptibles d'influencer tous les sous-systèmes de la société dans le sens d'une intégration de nouvelles «exigences» dans leurs programmes. Exigences susceptibles d'influencer à leur tour des décisions, certes, mais dont les prises sur le monde proprement dit demeurent amorties ${ }^{38}$. Selon cette approche, la généralisation des communications écologiques place l'ensemble des sous-systèmes de la société dans un état de résonance, sur lequel on ne peut se prononcer avec certitude quant aux résultats à l'échelle du système de la société dans son ensemble et de l'environnement.

En dépit de son caractère hermétique, la sociologie de Luhmann est compatible avec un nombre considérable de travaux consacrés à la perte d'influence de l'action à notre époque. Le succès de l'approche systémique et du paradigme de la gouvernance atteste de la pertinence de cette thèse. La gouvernance ${ }^{39}$ étant une traduction concrète de la perte de légitimité du politique ${ }^{40}$ et de la nécessité de transposer les modalités de l'action politique et publique dans des grammaires plus appropriées à ce nouvel état social.

Selon cette modélisation de l'action, l'agentivité de l'action humaine oscille entre quantité négligeable et impulsion déterminante, à l'image du battement d'aile du papillon qui déclenche un tsunami à l'autre bout de la planète. Un geste peut se diluer au point de s'effacer ou avoir un effet retentissant. Ces trajectoires étant davantage l'expression d'effets dominos que la concrétisation de stratégies bien pensées et menées, elle remettent en question le statut de l'acteur. On est en droit de se demander qui de l'acteur ou du système en sont in fine les auteurs ? À cet égard, on ne peut plus parler d'action, d'intervention, de décision au sens classique d'un agir raisonné, rationnel et stratégique. Les acteurs peuvent encore se penser comme tels et avoir des intentionnalités, mais ces dernières ne trouvent plus la place qui

37. Florence Rudolf, «Une sociologie de l'environnement sans contexte: Niklas Luhmann, une réception ambivalente», MANA, Caen, Presses Universitaires de Caen, $\mathrm{n}^{\circ}$ 12-13, 2005, p. 215-240. Estelle Ferrarese, Niklas Luhmann, une introduction, Paris, Pocket/La Découverte, 2007.

38. Florence Rudolf, «Une sociologie...», op. cit.

39. Jean-Pierre Gaudin, Pourquoi la gouvernance?, Paris, Presses de la FNSP, 2002.

40. À ce point concrète qu'elle procède des acteurs non politiques en quête de légitimité et de pouvoir pour faire advenir un monde multipolaire ou accélérer sa survenue. La Gouvernance. Dossier documentaire, www.hautsde-seine.net. 
étaient la leur dans les théories sociologiques classiques de l'action et de l'acteur ${ }^{41}$. Comment se représenter l'écologisation de la société dans une telle constitution sociale? Par-delà la question de la place de l'action individuelle et collective, propre à la sociologie classique, quel type de transformation est-on en mesure de se représenter? La faiblesse des images, des récits, voire l'absence de vision que nous avons soulignée à propos de ces discours, ne sont-elles pas tout simplement dues à ce que la figure même de société, au sens d'une entité sui generis, d'un corps social ou d'une entité intégrée ${ }^{42}$, comme elle s'impose encore au $\mathrm{XIX}^{\mathrm{c}}$ et au $\mathrm{XX}^{\mathrm{c}}$ siècle, a perdu de son sens?

À l'observation, l'incroyable résilience du système annoncée en début de paragraphe contraste avec les transformations auxquelles nous parvenons au terme de ce petit excursus consacré à l'homéostasie du système. Une autre figure se profile avec de plus en plus d'insistance, à savoir celle d'un effondrement brutal et sans précédent de mémoire d'hommes. Les sémantiques se ressemblent par les alertes qu'elles lancent, mais se distinguent surtout par la perspective du chaos qu'elles parviennent de moins en moins à masquer. La définition de la transition qu'Yves Luginbuhl, directeur de recherche CNRS émérite, choisit entre plusieurs options est peut-être la plus explicite à cet égard. Selon lui, la transition énergétique que nous vivons actuellement correspondrait à

une phase particulière de l'évolution d'une société, celle où elle rencontre de plus en plus de difficultés, internes ou externes, à reproduire le système économique et social sur lequel elle se fonde et commence à se réorganiser, plus ou moins vite et plus ou moins violemment sur la base d'un autre système qui, finalement, devient à son tour la forme générale des conditions nouvelles d'existence ${ }^{43}$.

La transition économique, dont il est si peu question dans toutes ces sémantiques qui se sont succédé depuis les années 1970, finirait-elle par s'imposer en raison d'un déni qui précipiterait son avènement? La thèse de l'effondrement, dont on ne sait plus trop s'il convient de s'en réjouir ou non, revient inexorablement sur le devant de la scène.

41. Cette sociologie «débute» avec le programme des sciences de la culture au XIXe siècle, programme que la sociologie allemande ne cessera d'incarner de Max Weber à Jürgen Habermas et Hans Joas, en passant par Georg Simmel. Bien que rassemblé autour du paradigme de l'action, ce programme se décline autour de postulats épistémologiques différenciés. Bien que le paradigme de l'action soit également représenté en France, autour des travaux d'Alain Touraine, notamment, ce n'est que tardivement et sur un mode mineur que l'approche par les significations sociales, le sens, les actions réciproques et la complexité accède à une certaine visibilité et audience en France. Les travaux de Patrick Watier, consacrés à la réhabilitation de Georg Simmel en France, et aux significations de l'action ont participé de cet effort. Patrick Watier, La sociologie et les représentations de l'activité sociale, Paris, Méridiens Klincksieck/Masson, 1996.

42. Bruno Latour, Changer la société, refaire de la sociologie, Paris, La Découverte, 2006.

43. Les futurs ateliers de recherche-action «Paysage, Territoires, Transitions», Note de cadrage 2015 - Document de travail, Ministère de l'Écologie, du Développement durable et de l'Énergie, p. 3. 
Qu'en est-il in fine de notre état social? Homéostasie versus effondrement? La première étant entretenue par le discrédit qui pèse sur l'action et l'engagement. Dans un état d'entre-capture systémique, dont il paraît peu probable de se sortir, et un monde fuyant qu'on ne sait par quel bout prendre, on ne peut exclure que cette indétermination imprègne la conscience des acteurs et par conséquent leurs manières de se représenter l'action. La préférence pour des investissements pragmatiques, soit pour des formes d'implication circonscrites à des petits gestes susceptibles de faire la différence pourrait se comprendre en référence à une dissolution de l'idée même de société. Conformément à cette proposition, l'engagement se concentrerait sur l'accompagnement de projets précis dont il est permis d'espérer des retours d'expériences encourageants, au sens d'un projet qui aboutit et débouche sur un autre et ainsi de suite...

Dans cette perspective, qui n'est pas sans rappeler la cité par projet ${ }^{44}$, on est assez proche d'une interprétation luhmanienne de la soutenabilité comme autopoièse systémique, structurée autour de la résilience. Cette conception est confortée par une nouvelle sémantique que nous aurions pu intégrer dans notre parcours, en raison du succès croissant qu'elle enregistre. Le seul bémol de cette nouvelle «idée régulatrice» de la modernité est qu'elle ne cherche même plus à se présenter comme un rempart contre la catastrophe et la crise, puisqu'elle nous invite à la vivre normalement. «Le vivre avec la crise» revient en effet comme un refrain dans les discours sur la résilience. Autant se préparer au pire.

\section{Conclusion}

Les appels à la nature ou à la vie invitent à considérer les rapports sociaux sous l'angle de leurs conséquences mortifères tant du point de vue anthropologique qu'écologique. La transition énergétique prend place dans une dynamique qui de la modernisation écologique au développement durable a profité à la substitution d'une version incrémentale du changement social au renversement de l'ordre social, hérité des luttes sociales du XIX' ${ }^{\mathrm{c}}$ siècle en quête d'un nouveau contrat social ${ }^{45}$. Ce nouvel imaginaire social, qualifié encore de pragmatique, a bénéficié de conjonctures économiques favorables, actuellement en panne. Dans quelle mesure la transition énergétique, dernière-née d'une longue série de communications sociales relayant la cause écologique, intègre-t-elle cette historicité? L'enquête non exhaustive sur les discours prononcés dans différentes assemblées et consignés dans des textes

44. Luc Boltanski, Eve Chiapello, Le nouvel esprit du capitalisme, Paris, Gallimard, 1999.

45. Jacques Donzelot, L'invention du social. Essai sur le déclin des passions politiques, Paris, Fayard, 1984. 
édités par différents collectifs au stade du projet de loi sur la transition énergétique conforte la thèse selon laquelle plusieurs décennies de mobilisations sociales, au sens d'une production multipolaire de sémantiques ou communications sociales qui lancent l'alerte écologique et maintiennent la pression sur le système de la société, permettent de douter d'une inflexion douce de la modernité. La formule consacrée selon laquelle la transition énergétique correspond au passage d'un état à un autre, sans plus de précision, est riche de sens. Elle témoigne d'une impasse qui se resserre et se précise autour de la thématique de l'effondrement. Il ne s'agit même plus de préservation du monde mais de dresser un dernier rempart contre le chaos. Cette alternative génère une situation d'hébètement et de fascination aux effets de prophétie autoréalisatrice pervers au sens où on peut la suspecter de précipiter un effondrement généralisé. Orphelins des visions et images qui guidaient notre rapport au monde, nous assistons impuissants à la catastrophe qui vient ou, au mieux, nous nous préparons à y faire face ${ }^{46}$ en vue de l'après et de la reconstruction post-catastrophe ${ }^{47}$.

46. Jean-Pierre Dupuy, Pour un catastrophisme éclairé, Paris, Seuil, 2002. Isabelle Stengers, Au temps des catastrophes. Résister à la barbarie qui vient, Paris, La découverte, 2009. Erik M. Conway, Naomi Oreskes, L'effondrement de la civilisation occidentale, Lonrai, Édition Les liens qui libèrent, 2014.

47. Battlefield, une pièce de Peter Brook, trente ans après la création de son Mahabharata, Théâtre des Bouffes du Nord, Paris, septembre-octobre 2015, www.newspeterbrook.com. 